OPEN ACCESS

Edited by:

Michael Levy,

Massachusetts General Hospital,

United States

Reviewed by:

Itay Lotan,

Rabin Medical Center, Israel

Silvia Noemi Tenembaum,

Garrahan Hospital, Argentina

*Correspondence: Kevin Rostásy

k.rostasy@kinderklinik-datteln.de

Specialty section:

This article was submitted to

Neuroepidemiology,

a section of the journal

Frontiers in Neurology

Received: 15 March 2020

Accepted: 20 April 2020

Published: 15 May 2020

Citation:

Lechner C, Breu M, Wendel E-M, Kornek B, Schanda K, Baumann M,

Reindl $M$ and Rostásy K (2020) Epidemiology of Pediatric NMOSD in

Germany and Austria.

Front. Neurol. 11:415.

doi: 10.3389/fneur.2020.00415

\section{Epidemiology of Pediatric NMOSD in Germany and Austria}

\author{
Christian Lechner ${ }^{1}$, Markus Breu ${ }^{2}$, Eva-Maria Wendel ${ }^{3}$, Barbara Kornek ${ }^{4}$, \\ Kathrin Schanda ${ }^{5}$, Matthias Baumann ${ }^{1}$, Markus Reindl ${ }^{5}$ and Kevin Rostásy ${ }^{*}$
}

${ }^{1}$ Division of Pediatric Neurology, Department of Pediatrics I, Medical University of Innsbruck, Innsbruck, Austria, ${ }^{2}$ Department of Pediatrics and Adolescent Medicine, Medical University of Vienna, Vienna, Austria, ${ }^{3}$ Department of Pediatrics, Olgahospital Stuttgart, Stuttgart, Germany, ${ }^{4}$ Department of Neurology, Medical University of Vienna, Vienna, Austria, ${ }^{5}$ Department of Neurology, Medical University of Innsbruck, Innsbruck, Austria, ${ }^{6}$ Department of Pediatric Neurology, Children's Hospital Datteln, University Witten/Herdecke, Datteln, Germany

Background: Neuromyelitis optica spectrum disorders (NMOSD) are severe inflammatory demyelinating disorders of the central nervous system mainly characterized by recurrent episodes of uni- or bilateral optic neuritis (ON), transverse myelitis (TM) and brainstem syndromes (BS). The majority of adult patients has serum antibodies directed against the water channel protein aquaporin 4 (AQP4-abs). In pediatric patients, AQP4-abs are less, while antibodies against myelin oligodendrocyte glycoprotein (MOG-abs) are more frequently detectable than in adults. Some children with NMOSD have neither AQP4- nor MOG-ab (double-seronegative).

Objective: Evaluation of epidemiological data regarding incidence and prevalence of pediatric NMOSD in Germany and Austria.

Methods: We recruited pediatric NMOSD patients between 1 March 2017 and 28 February 2019 with five different tools: (1) ESPED (Surveillance Unit for Rare Pediatric Disorders in Germany), (2) ESNEK (Surveillance for Rare Neurological Disorders during Childhood), (3) pediatric neurology working group within the Austrian Society of Pediatrics and Adolescent Medicine, (4) BIOMARKER Study and (5) NEMOS (Neuromyelitis optica Study Group). We requested data regarding clinical symptoms, antibody status, therapy regimen and response via a standardized questionnaire.

Results: During the 2-year recruitment period, 46 (both incidental and prevalent) patients with a suspected diagnosis of NMOSD were brought to our attention. Twenty-two of these patients did not fulfill the inclusion criteria. Of the remaining 24 children, 22 had a median age at onset of 11 (range 3-17) years and 16/22 were female (72.7\%) (no data in two patients). Sixteen of 24 patients were AQP4-ab positive (67\%), 4/24 MOG-ab positive (16.7\%), three children were double-seronegative and in one patient no antibody testing was done. We calculated an incidence rate of 0.022 per 100,000 person-years for Germany, while there was no incidental case in Austria during the recruitment period. The prevalence rate was 0.147 and 0.267 per 100,000 persons in Germany and Austria, respectively. 
Conclusion: Pediatric NMOSD, with and without associated antibodies, are very rare even considering the different limitations of our study. An unexpected finding was that a considerable proportion of patients was tested neither for AQP4- nor MOGabs during diagnostic work-up, which should prompt to establish and disseminate appropriate guidelines.

Keywords: NMOSD, AQP4-antibodies, MOG-antibodies, transverse myelitis, optic neuritis, brainstem syndrome

\section{INTRODUCTION}

Neuromyelitis optica spectrum disorders (NMOSD) are severe inflammatory demyelinating disorders of the central nervous system mainly characterized by simultaneous or sequential episodes of uni- or bilateral optic neuritis (ON), transverse myelitis (TM) and brainstem syndromes (BS) $(1,2)$. The discovery of a specific autoantibody in NMO patients, targeted against the water channel protein aquaporin 4 (AQP4) located in high density in astrocytic processes at the blood-brain barrier, in 2004 supported the differentiation between NMO and MS (3). In the following, multiple studies could show that AQP4antibodies (AQP4-abs) are detectable in up to $80 \%$ of adult patients diagnosed with $\mathrm{NMO}(4-6)$. This led to a modification of the diagnostic criteria for NMO by adding the presence of AQP4abs as a supportive criterion (7-10). Increasing AQP4-abs assay quality and reliability as well as further publications showing the connection between AQP4-abs and different clinical phenotypes other than ON and TM, resulted in another revision of the diagnostic criteria and led to the extension of NMO utilizing the umbrella term NMOSD (11-13).

Up to $20 \%$ of adult NMOSD patients remain AQP4-antibody (ab) negative (14-18) and a certain proportion of these AQP4-ab negative patients show antibodies against myelin oligodendrocyte glycoprotein (MOG-abs) (19-23). Recent studies showed that pediatric and adult NMOSD patients with MOG-abs can also have recurrent disease courses (24-28). The reported prevalence of MOG-abs in AQP4-ab negative pediatric patients shows a wide range between different working groups, possibly due to different inclusion criteria and unreported MOG$\mathrm{ab}$ status. Consequently, in some studies the majority of NMOSD patients show MOG-abs while others report similar frequencies of AQP4-abs in pediatric as in adult patients (23, 29-32).

NMOSD is, by definition of $\mathrm{WHO}$ and $\mathrm{EU}$, considered a rare disease with 1 (or fewer) in 2,000 individuals affected. Several population-based studies, focussing primarily on adult patients,

\footnotetext{
Abbreviations: ab, antibody; abs, antibodies; ADS, acquired demyelinating syndrome; APS, area postrema syndrome; AQP4-abs, antibodies against aquaporin 4; AZA, azathioprine; bilON, bilateral optic neuritis; BS, brainstem syndromes; DMF, dimethyl fumarate; DMT, disease-modifying therapy; ESNEK, Rare paediatric neurological disease registry Germany; ESPED, Surveillance Unit for Rare Paediatric Disorders in Germany; GLAT, glatiramer acetate; IVIG, intravenous immunoglobulins; IVMP, intravenous methylprednisolone; LETM, longitudinally extensive transverse myelitis; MMF, mycophenolate mofetil; MOG-ab, antibodies against myelin oligodendrocyte glycoprotein; NMOSD, neuromyelitis optica spectrum disorders; OCB, oligoclonal bands; ÖGKJ, Austrian Society of Paediatrics and Adolescent Medicine; ON, optic neuritis; PLEX, plasma exchange; pos, positive; RTX, rituximab; TM, transverse myelitis; unilON, unilateral optic neuritis.
}

showed incidence rates of 0.053 to 0.4 per 100,000 person-years and prevalence rates of 0.52 to 4.4 per 100,000 people (33-39). However, none of these studies applied the revised diagnostic criteria of 2015 and thus it remains unknown if (and how much) the incidence and prevalence rates were affected by the broadening of the spectrum. So far, Sepúlveda et al. 2015 criteria increased incidence and prevalence by 1.5 times (18). Hyun et al. even reported a 1.85 -fold increase (40).

There are only a few studies focusing on the frequency of acquired demyelinating syndromes (ADS), among them NMOSD, in children (41-43). Very recently Boesen et al. specifically undertook a population-based, multicentre cohort study to estimate the incidence of pediatric NMOSD in Denmark, which was 0.031 per 100,000 person-years (44). An Australian single-center retrospective study could show that five of 67 (7.5\%) pediatric patients presenting with ADS between 2007 and 2014 were diagnosed with NMOSD (43).

The aim of our study was to ascertain the incidence and prevalence of pediatric NMOSD in Germany and Austria, using the 2015 criteria. Subsequently, we evaluated if these patients would also fulfill the 2006 criteria and calculated the respective incidence and prevalence rates.

\section{MATERIALS AND METHODS}

\section{Setting}

Germany and Austria are two geographically and politically defined countries located in Western and Middle Europe, respectively, with a combined area of $441,265 \mathrm{~km}^{2}$. Austria has 8,851,417 (30 October 2018 census) and Germany 83,019,213 (31 December 2018 census) inhabitants, resulting in a total of $91,870,630$ people. By information of the Federal Offices of Statistics (Statistik Austria and Statistisches Bundesamt Deutschland) 1,535,958 and 13,597,428 respectively (combined $15,133,386)$, of these $91,870,630$ inhabitants are underage (16.5\%). Both countries have a majority of Caucasian ethnicity, however to our knowledge the official census institutions do not collect the population's ethnicities. Health care in Austria and Germany is provided by an open access public health care system with a network of pediatric neurologists specializing on demyelinating disorders.

\section{Case Ascertainment Tools and Study Populations}

For this clinic- and questionnaire-based multicentre pro- and retrospective study we used the following tools to estimate the incidence and prevalence of pediatric NMOSD between 1 March 2017 and 28 February 2019: 
1) We initiated a prospective epidemiological study via ESPED (Surveillance Unit for Rare Pediatric Disorders in Germany, in German "Erhebungseinheit für $\underline{\text { Seltene }}$ Pädiatrische Erkrankungen in Deutschland"; based in Dusseldorf) and included incidental cases of pediatric NMOSD diagnosed during the investigation period. Every pediatric department in Germany has one responsible colleague designated to report patients with newly-diagnosed rare diseases to the different ongoing ESPED studies.

2) Furthermore, we used ESNEK (Surveillance for Rare Neurological Disorders during Childhood, in German "Erhebung seltener neurologischer Erkrankungen im Kindesalter"; based in Göttingen) as an e-mail-based recruitment tool once a year during our recruitment period. Via ESNEK, we contacted all $\sim 1,200$ members of the Society for Neuropediatrics (Gesellschaft für Neuropädiatrie) and by this the majority of all Germany- and Austria-based pediatric neurologists and asked to report pediatric NMOSD patients, diagnosed and/or under their care during the recruitment period.

3) By using the e-mail distribution list of the pediatric neurology working group within the Austrian Society of Pediatrics and Adolescent Medicine, we contacted about 170 (and thereby most) Austria-based pediatric neurologists and asked to support our epidemiological study by referring pediatric NMOSD patients using our standardized questionnaire. As the majority of these colleagues are also part of the Society for Neuropediatrics, they received our e-mails twice each time.

4) Additionally, we included all pediatric NMOSD patients who were referred to our BIOMARKER study (based in Innsbruck and Datteln) between March 2017 and February 2019. This is an ongoing prospective, multicentre study started in 2009 with currently more than 900 included children and teenagers presenting with the first event of an ADS. We also included all previously referred children with ongoing followups who fulfilled the inclusion criteria and were still $<18$ years old.

5) Finally, we contacted the adult NMO Study Group, called NEMOS, currently with representatives in 45 neurology clinics in Germany and asked how many pediatric (both incidental and prevalent) NMOSD patients had been brought to their attention between March 2017 and February 2019 and had been included in their patient registry. Representatives from about 25 German universities founded NEMOS in 2008 to improve the care of NMOSD patients (45).

\section{Study Population and Diagnostic Criteria}

Patients included in this study had to meet the following inclusion criteria: (1) diagnosis of NMOSD fulfilling the 2015 International Panel for NMO Diagnosis criteria (12), (2) age below 18 years at disease onset, and (3) written informed consent. Exclusion criteria included the diagnosis of another type of ADS like MS or an infectious, metabolic, vascular, or neoplastic CNS disease.

\section{Standard Protocol Approvals, Registrations, and Patient Consents}

The study was approved by the Ethics Committee of the Medical University of Innsbruck, Austria (Study number AN4059) and by the Ethics Committee of the Witten/Herdecke University, Germany (Study number 10/2017). All patients and/or their caregivers provided written informed consent.

\section{Antibody Assays}

Serum samples were analyzed for the presence of MOG- and AQP4-abs by live cell-based immunofluorescence assays as previously described $(46,47)$.

Using the above-mentioned case ascertainment tools, not all serum samples were tested in Innsbruck. Serum of patients recruited via ESPED was not referred to our lab in Innsbruck and thus screened for MOG- and AQP4-abs by unknown assays. However, it is very likely that AQP4ab testing was either done with a live cell-based assay (CBA) or with the well-evaluated Euroimmun kit (13). MOG-ab testing is possible with an Euroimmun kit as well, though the sensitivity and specificity are not as high as established live CBAs (47). Treating doctors might have sent serum samples to neuroimmunology labs in Heidelberg (S. Jarius) or in Kiel (F. Leypoldt), which both use the above-mentioned live CBAs.

\section{Statistical Methods}

All of the $95 \%$ confidence intervals (95\% CI) were calculated for the prevalence and incidence rate estimates using the modified Wald method. Quantitative variables were described using median and range. Statistical analysis was performed using IBM SPSS, release V.24.0 (IBM Corporation).

\section{RESULTS}

With the above-mentioned ascertainment tools, a total of 46 pediatric patients with a suspected diagnosis of (both incidental and prevalent) NMOSD were brought to our attention. In $40 / 46$ children we had enough clinical information necessary to evaluate the patients' diagnoses using the 2015 criteria. We identified 22 patients who did not fulfill the inclusion criteria and were thus excluded from this study (see Figure 1). None of the excluded patients fulfilled the 2006 criteria. All of these excluded patients were recruited via ESPED, meaning they were referred anonymously. Therefore, the type of antibody assay, used in $18 / 22$ patients, was unknown. The remaining four patients were not tested for MOG- and AQP4-abs at all.

Of the remaining 24 children, 22 had a median age at onset of 11 (range $3-17$ ) years and 16/22 were female (72.7\%) (no data in two patients). In $18 / 24$ patients, ethnicity was reported: $13 / 18$ were Caucasian (72\%), 3/18 (16.7\%) were from the Near or Middle East or Egypt, 1/18 (6\%) was African and 1/18 (6\%) from South Asia.

Sixteen of 24 patients were AQP4-ab positive (67\%), 4/24 MOG-ab positive (16.7\%), three children were MOG- and AQP4ab negative (double-seronegative) and in one patient no antibody testing was done. None of these patients were positive for both 


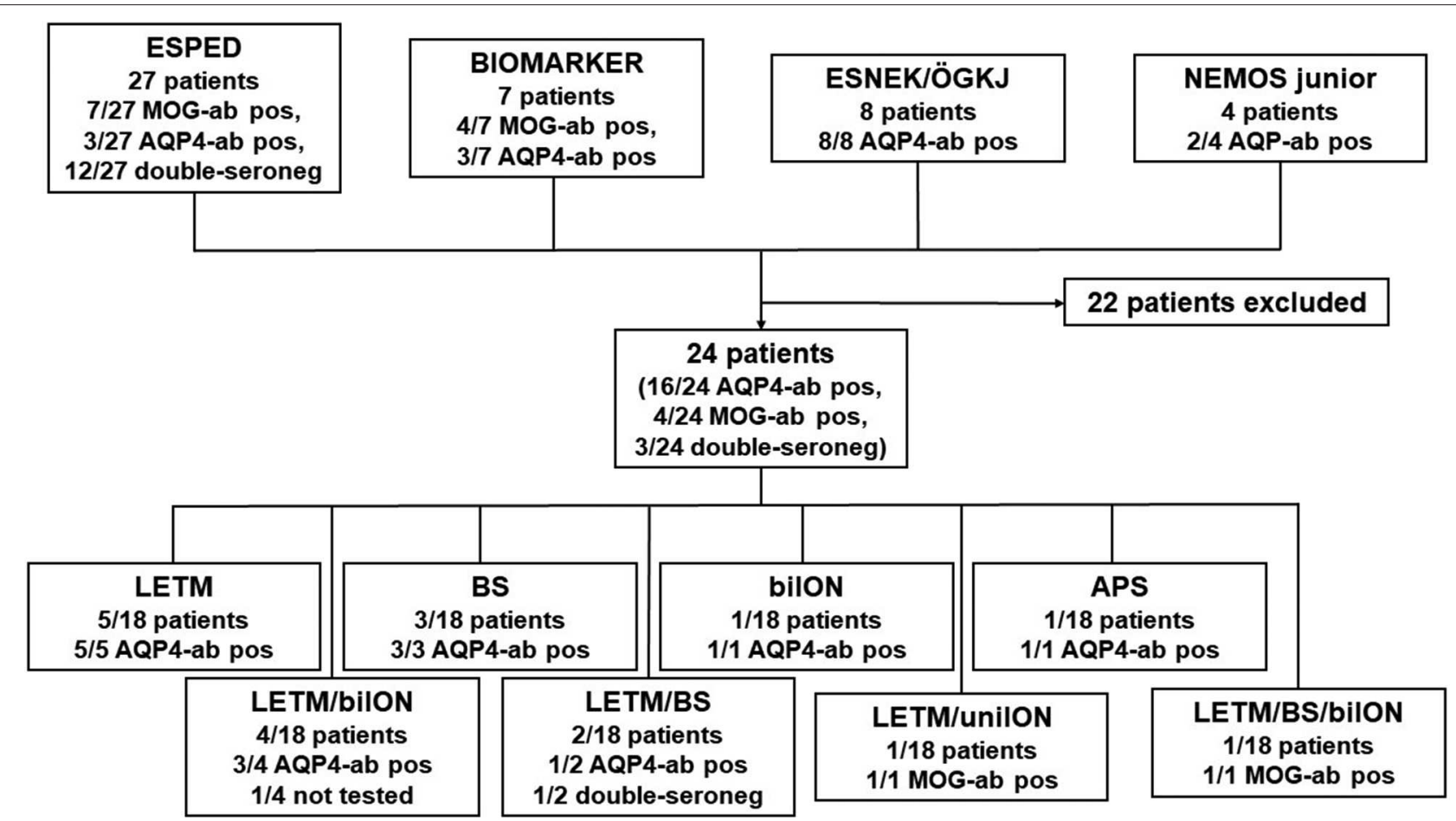

FIGURE 1 | Flow chart study profile. APS, area postrema syndrome; AQP4-ab, antibodies against aquaporin 4; bilON, bilateral optic neuritis; BS, brainstem syndromes, ESNEK, Surveillance for Rare Neurological Disorders during Childhood; ESPED, Surveillance Unit for Rare Paediatric Disorders in Germany; LETM, longitudinally extensive transverse myelitis; MOG-ab, antibodies against myelin oligodendrocyte glycoprotein; ÖGKJ, Austrian Society of Paediatrics and Adolescent Medicine; unilON, unilateral optic neuritis.

MOG- and AQP4-abs. In 19/23 patients, antibody testing was done with a live CBA. Thirteen of these 19 patients were screened in Innsbruck. The remaining four patients were recruited via ESPED and due to its anonymous referral method, we could not ask treating doctors which assay was used. However, as described above, it is very likely that well-established assays or kits were used.

Detailed clinical data was available for 18/24 patients (see Figure 1 and Supplementary Table 1). All 18 patients received intravenous methylprednisolone (IVMP) as acute therapy. Additional treatments at onset included intravenous immunoglobulins (IVIG; $n=7$; 4/7 AQP4-ab pos, 1/7 MOG-ab pos, $1 / 7$ double-seronegative, $1 / 7$ not tested), plasma exchange (PLEX; $n=7 ; 5 / 7$ AQP4-ab pos, 1/7 double-seronegative, 1/7 not tested) and rituximab (RTX; $n=2 ; 2 / 2$ AQP4-ab pos). $14 / 18$ pediatric patients were subsequently started on long-term treatments: RTX ( $n=8 ; 1 / 8$ after relapses on azathioprine; $7 / 8$ AQP4-ab pos, $1 / 8$ double-seronegative), azathioprine (AZA; $n=$ 4; 4/4 AQP4-ab pos), tocilizumab ( $n=2$; after relapses on RTX; 2/2 AQP4-ab pos), mycophenolate mofetil (MMF; $n=1$; not tested for autoantibodies), IVIG $(n=1)$ and cyclophosphamide ( $n=1$; after relapses on RTX; 1/1 AQP4-ab pos). We had no information about the type of DMT in one patient.

Of the remaining six patients without clinical information, four were referred via NEMOS with a confirmed diagnosis of NMOSD. Two of these four patients were AQP4-ab positive.
The other two patients (not referred via NEMOS), both AQP4ab positive, were brought to our attention as NMOSD without further details.

Further demographic and clinical details of these patients are summarized in Table 1 and Supplementary Table 1.

\section{Study Populations ESPED}

During our observation period, a total of 27 pediatric patients were referred via ESPED with a diagnosis of NMOSD. However, 22 of these 27 patients $(81.5 \%)$ with a median age at diagnosis of 14 (range 2-17) years, did not meet the diagnostic criteria (neither the 2006 nor the 2015): Seven of these 22 patients showed MOG-abs, 11 were tested negative for AQP4- and MOG-abs and in four no antibody testing was done. Clinically, 11 presented with ON, six with TM, three with LETM, and two with simultaneous TM (meaning less than three involved segments on spinal MRI) and ON. All these 22 pediatric patients were excluded.

The remaining five patients fulfilled the inclusion criteria. AQP4-abs were detected in $3 / 5$ children $(3$ males, median age 7 [range 4-11] years), while one 13 year-old male patient was double-seronegative (patient 14) and in one 9 year-old female patient no antibody testing was done as she had already received IVIG prior to sample collection (patient 3). The cumulative median age was nine (range $4-13$ ) years, 4/5 patients were male. 
TABLE 1 | Demographic and clinical characteristics of the included patients.

\begin{tabular}{|c|c|}
\hline & Patients $(n=24)$ \\
\hline Female sex, n (\%) & 16/22 (72.7\%) \\
\hline Ratio female:male & $2.67: 1$ \\
\hline \multicolumn{2}{|l|}{ Ethnicity, n (\%) } \\
\hline Caucasian & 13/18 (72\%) \\
\hline Near or Middle East or Egypt & 3/18 (16.7\%) \\
\hline African & $1 / 18(6 \%)$ \\
\hline South Asia & $1 / 18(6 \%)$ \\
\hline \multicolumn{2}{|l|}{ Antibody status, n (\%) } \\
\hline AQP4-abs & $16 / 24(67 \%)$ \\
\hline MOG-abs & $4 / 24(16.7 \%)$ \\
\hline Double-seronegative & $3 / 24(12.5 \%)$ \\
\hline Not tested & $1 / 24(4.2 \%)$ \\
\hline Age at onset (years), median (range) & $11(3-17)$ \\
\hline \multicolumn{2}{|l|}{ Clinical attack at onset, $n$ (\%) } \\
\hline Longitudinally extensive transverse myelitis & $5 / 18(27.8 \%)$ \\
\hline Bilateral optic neuritis + LETM & $4 / 18(22.2 \%)$ \\
\hline Brainstem syndrome & 3/18 (16.7\%) \\
\hline LETM + brainstem syndrome & 2/18 (11.1\%) \\
\hline Bilateral optic neuritis & $1 / 18(5.6 \%)$ \\
\hline Unilateral optic neuritis + LETM & $1 / 18(5.6 \%)$ \\
\hline Area postrema syndrome & $1 / 18(5.6 \%)$ \\
\hline Bilateral ON + LETM + BS & $1 / 18(5.6 \%)$ \\
\hline \multicolumn{2}{|l|}{ Cerebral MRI, n (\%)* } \\
\hline Normal & 5/18 (27.8\%) \\
\hline Non-specific WM lesions & 6/18 (33.3\%) \\
\hline Brainstem involvement & $5 / 18(27.8 \%)$ \\
\hline Optic nerves involvement & 2/18 (11.1\%) \\
\hline \multicolumn{2}{|l|}{ Spinal MRI, n (\%)* } \\
\hline Normal & $5 / 18(27.8 \%)$ \\
\hline LETM & $13 / 18(72.2 \%)$ \\
\hline TM & 0/18 (0\%) \\
\hline \multicolumn{2}{|l|}{ Acute therapy, n (\%) } \\
\hline Intravenous methylprednisolone & 18/18 (100\%) \\
\hline Add-on therapy (IVIG, etc.) & $8 / 18(44.4 \%)$ \\
\hline Long-term therapy, n (\%) & 14/18 (77.8\%) \\
\hline
\end{tabular}

"The minimum requirements to re-evaluate the referred MRI results were available imaging data with (contrast-enhanced) T1 and T2 for the spinal MRI and (contrast-enhanced) T1, T2, and FLAIR for the cerebral MRI. These criteria were fulfilled by 12/24 patients.

Clinically, one AQP4-ab positive patient presented with an area postrema syndrome (patient 1), two with a simultaneous LETM and bilON (1 AQP4-ab positive patient and patient 3) and two with a simultaneous LETM and BS (1 AQP4-ab positive patient and patient 14).

Four of five patients did not only receive IVMP as acute treatment, but also at least one of the following: PLEX $(n=4)$, IVIG $(n=3)$ and RTX $(n=2)$. These four children were also put on long-term treatments: $\operatorname{RTX}(n=3)$ and $\operatorname{MMF}(n=1)$.

\section{ESNEK and Pediatric Neurology Working Group Within the Austrian Society of Pediatrics and Adolescent Medicine}

By contacting the majority of the Germany- and Austria-based pediatric neurologists via e-mail, seven so far unknown patients were brought to our attention. Another patient was already part of our BIOMARKER Study but reported again via ESNEK.

All these eight patients were AQP4-ab positive. Two patients were referred only stating their antibody status and without further demographic or clinical details. The remaining six patients had a median age of 13 (range 10-17) years and all of them were females. Interestingly, 5/6 patients initially presented with LETM. The remaining patient (patient 29) had a BS.

All patients were given IVMP, 3/6 additionally received IVIG and PLEX. Regarding the long-term treatment, we had no information about one patient (patient 39). Two of the remaining five children received the IL-6-receptor antagonist tocilizumab (after relapses on RTX), 1/5 azathioprine (AZA), 1/5 RTX and one patient did not respond to RTX and was changed to cyclophosphamide.

\section{BIOMARKER Study}

Since 2009, more than 900 children with a first (suspected) event of ADS were referred to our BIOMARKER Study and tested for MOG- and AQP4-abs. Within this cohort, seven patients fulfilled the diagnostic criteria and were still underage at the beginning of our observation period.

Four of seven patients were MOG-ab positive, 3/7 AQP4-ab positive. Their median age was 9 (range 3-14) years, 5/7 patients were female. One AQP4-ab positive, female patient (patient 33) had an $\mathrm{ON}$ as onset attack, the other two AQP4-ab positive children presented with a BS. The remaining four MOG-ab positive patients had simultaneous ON and LETM.

Every patient was treated with IVMP during the clinical event. Only one female, MOG-ab positive teenager (patient 43) additionally received IVIG. The three AQP4-ab positive patients were started on AZA and one was changed to RTX due to insufficient therapy response. Among the MOG-ab positive patients, only one received IVIG as long-term treatment (patient 44).

\section{NEMOS}

By contacting NEMOS and asking to report NMOSD patients who were underage at the beginning of our observation period, we could include four additional patients into this study, who have not been identified with one of the other tools.

Two of these patients were AQP4-ab positive and the other two AQP4-ab negative. In the latter two MOG-ab status was unknown. The median age was 11 (range 6-16) years, all four patients were females. One AQP4-ab negative patient (patient 34) was newly-diagnosed during our observation period and was added to the incidental cases. Besides age, sex and antibody status we did not receive any further demographic or clinical details.

\section{Incidence and Prevalence}

Overall, six Germany-based patients were newly-diagnosed with NMOSD during our observation period and thus considered as incidental cases. Within our 2-year recruitment period, no child was newly-diagnosed with NMOSD in Austria. The remaining 18 children had already been diagnosed prior to our recruitment period and were therefore categorized as prevalent cases. 
Considering a total of $13,597,428$ minors in Germany, we estimated an incidence rate of 0.022 (95\% CI $0.005-0.066$ ) per 100,000 person-years. If we included only the three AQP4-ab positive patients, the estimated incidence rate was 0.011 (95\% CI 0.002-0.033) per 100,000 person-years. For Austria, lacking an incidental case, we could not calculate the incidence rate.

With 20 pediatric NMOSD patients, the estimated prevalence rate in Germany was 0.147 (95\% CI 0.096-0.217) per 100,000 persons, considering only the 12 AQP4-ab positive patients, it was 0.088 per 100,000 (95\% CI $0.0456-0.154)$. Doubleseronegative pediatric NMOSD $(n=3)$ had a prevalence of 0.022 (95\% CI 0.005-0.066) per 100,000, and the four MOG-ab positive patients, fulfilling the diagnostic criteria for NMOSD, $0.029(95 \%$ CI $0.009-0.076)$ per 100,000 . One of these 20 patients was not tested for MOG- or AQP4-abs (patient 3).

In Austria, considering a total of 1,535,958 minors and four reported pediatric AQP4-ab positive NMOSD patients, the prevalence rate was 0.267 (95\% CI 0.105 to 0.524$)$ per 100,000 persons.

If we apply the 2006 criteria, only four of the above mentioned, Germany-based six patients would count as incidental cases, with one of them being AQP4-ab positive. Respectively, the incidence rate would be 0.015 ( $95 \%$ CI 0.001 to 0.055 ) cases per 100,000 person-years, resulting in an $\sim 1.5$-fold increase if the 2015 criteria are used.

The prevalence rates would decrease to 0.118 (95\% CI $0.073-$ 0.184 ) per 100,000 persons in Germany and to 0.200 (95\% CI 0.064-0.460) per 100,000 persons in Austria.

\section{DISCUSSION}

Using different case ascertainment tools to assess and calculate the incidence and prevalence rate of pediatric NMOSD in Germany and Austria, we detected only 24 pediatric patients who fulfilled the 2015 criteria of NMOSD during our 2-year observation period. Nevertheless, the estimated incidence rate of 0.022 per 100,000 person-years (95\% CI: $0.0081-0.048$ ) is comparable to the systematic registry-based incidence rate of 0.031 per 100,000 person-years (95\% CI: $0.011-0.082$ ) calculated in the study by Boesen et al. (44) with both confidence intervals overlapping.

We could further show that a significant number of referred cases did not fulfill the 2015 NMOSD criteria, indicating that pediatric neurologists without special expertise in pediatric neuroimmunology are not familiar with the recently revised criteria.

In total, 22/27 pediatric patients (81.5\%) referred via ESPED were incorrectly classified as NMOSD. Our ESPED inquiry also revealed that in five patients ( $4 / 5$ were excluded from this study) no antibody testing was done at all. As antibody status could have had important diagnostic and therapeutic implications considering the disabling potential of relapses in AQP4-ab positive NMOSD, we strongly encourage pediatric neurologists to screen for MOG- and AQP4-abs in pediatric patients with ADS $(32,48)$. Furthermore, this lack of information should lead to the creation of better guidelines, facilitating diagnosis and therapy of pediatric ADS patients, which should be disseminated among physicians caring for children in particular with NMOSD.

Theoretically, it could have been possible that treating doctors referred their patients to us not only via ESPED, but also via ESNEK. As ESPED patients are reported anonymously, there is a chance that we counted double-referred patients twice and by this, created a falsely increased prevalence rate. However, postcodes of all ESPED reported patients were available and were double-checked with the patients referred via ESNEK or the BIOMARKER Study. The same issue arose with the four patients who were included via NEMOS. For these patients, we compared available data (age and antibody status) with the remaining patients and could thereby exclude that they had already been brought to our attention by another ascertainment tool.

Using both the 2006 and 2015 criteria, we could demonstrate that implementing the 2015 criteria has increased the incidence rate in Germany by 1.5 times. Similar increases of the incidence rate were also shown in recent studies [1.5 times in Sepulveda et al. (18), 1.85 times in Hyun et al. (40)].

Another important issue is the heterogeneity of NMOSD in the subgroup of AQP4-ab negative patients either harboring MOG-abs or being double-seronegative, which we included both in this epidemiological study. However, considering for example the different pathogenic mechanisms in AQP4-ab positive NMOSD (astrocytopathy) and MOG-ab associated disease (MOG-AD; oligodendrocytopathy), we calculated the prevalence both for AQP4- and MOG-ab positive patients separately fulfilling the 2015 criteria for NMOSD (0.088 vs. 0.029 per 100,000 persons, respectively). Appreciating the pathophysiological, clinical and radiological differences between AQP4-ab positive NMOSD and MOG-AD (also MOG-ab disease, MOG-encephalomyelitis, MOG spectrum disorders) it seems reasonable in the future to not include MOG-ab positive patients with a clinical phenotype of NMOSD, but to consider them as a separate disease entity $(17,20,21,25,28,49-56)$. While the pathophysiology of the double-seronegative NMOSD patients is still not understood, the clinical management remains the same, so we kept these patients in our calculation.

Sixteen of $24(67 \%)$ pediatric patients showed AQP4-abs, while only four patients had MOG-abs. These results are partly supported by the literature (29-31, 57-59). An explanation for the large proportion of AQP4-ab positive patients in our study might be the fact that awareness among physicians for NMOSD is especially high when their patients are tested positive for AQP4-abs. Still, children clinically presenting with ADS rather show MOG- than AQP4-abs (60). However, more than half of all MOG-ab positive patients remain monophasic and are thus unlikely to fulfill the 2015 diagnostic criteria for NMOSD (27, 61). MOG-ab positive patients fulfilling these criteria may not have been referred to our study due to the treating physicians' decision to classify these patients as MOG-AD.

Our study has the following strengths: (1) first study addressing the epidemiology of pediatric NMOSD in Germany and Austria, (2) usage of multiple ascertainment tools, and (3) available clinical data for $18 / 24$ patients (despite focussing on epidemiological data). 
However, several limitations need to be addressed: Specific registries, using medical data provided by insurances or the health care system, for pediatric NMOSD patients exist neither in Germany nor Austria. Accordingly, population-based studies are currently not possible in these countries. However, by using various tools to recruit patients, we tried to compensate for this limitation as much as possible.

We are aware of the fact that this study still has a certain selection bias as the referring colleagues do not represent the majority of all pediatric neurologists in Germany or Austria. For example, there is not a single patient referred from Berlinor Hamburg-based tertiary care children's hospitals, which is very unlikely considering that these are the two biggest cities in Germany. Therefore, we assume that there is a certain proportion of pediatric patients with NMOSD who were not reported to one of our ascertainment tools.

Another limitation is that we are not aware of the type of assay used in four patients referred via ESPED and in 18/22 excluded patients. However, it is very likely that well-established live CBA or commercially available Euroimmun kits were used to screen for MOG- and AQP4-abs.

In 6/24 included patients, we did not have sufficient clinical data to verify the referral diagnosis NMOSD. However, 4/6 patients were AQP4-ab positive making the diagnosis rather easy, and the two AQP4-ab negative patients were referred by an NMOSD expert consortium convincing us of the diagnosis.

\section{CONCLUSION}

Pediatric NMOSD, both with and without associated antibodies, are very rare disease entities. An unexpected finding was that a considerable proportion of patients was tested neither for AQP4- nor MOG-abs during diagnostic work-up, which should prompt to create and disseminate commonly available and easy-to-follow guidelines. Finally, we are convinced that multicentric studies with higher patient numbers are needed to evaluate the true epidemiology, long-term outcome and prognosis of pediatric patients with AQP4-ab positive and double-seronegative NMOSD as well as MOG-AD.

\section{REFERENCES}

1. Devic E. Myélite Aiguë Dorso-Lombaire Avec Névrite Optique. Autopsie. Congres Français de Médecine (Premiere Session; Lyon, 1894; procès-verbaux, mémoires et discussions) Lyon: Dr L. Bard (1895). p. 434-9.

2. Jarius S, Wildemann B. The history of neuromyelitis optica. J Neuroinflammation. (2013) 10:8. doi: 10.1186/1742-2094-10-8

3. Lennon VA, Wingerchuk DM, Kryzer TJ, Pittock SJ, Lucchinetti CF, Fujihara K, et al. A serum autoantibody marker of neuromyelitis optica: distinction from multiple sclerosis. Lancet. (2004) 364:2106-12. doi: 10.1016/s0140-6736(04)17551-x

4. Weinshenker BG, Wingerchuk DM, Vukusic S, Linbo L, Pittock SJ, Lucchinetti CF, et al. Neuromyelitis optica IgG predicts relapse after longitudinally extensive transverse myelitis. Ann Neurol. (2006) 59:566-9. doi: 10.1002/ana.20770

5. Wingerchuk DM. Neuromyelitis optica. Int MS J. (2006) 13:42-50.

\section{DATA AVAILABILITY STATEMENT}

All datasets generated for this study are included in the article/Supplementary Material.

\section{ETHICS STATEMENT}

The studies involving human participants were reviewed and approved by Ethics Committee of the Medical University of Innsbruck, Austria Ethics Committee of the Witten/Herdecke University, Germany. Written informed consent to participate in this study was provided by the participants' legal guardian/next of kin. Written informed consent was obtained from the minor(s)' legal guardian/next of kin for the publication of any potentially identifiable images or data included in this article.

\section{AUTHOR CONTRIBUTIONS}

$\mathrm{CL}$ and $\mathrm{KR}$ created concept and design of the study, jointly acquired, and analyzed the data. CL drafted the manuscript. MR supported the data analysis. MBr, E-MW, MBa, BK, KS, and MR acquired the data and critically reviewed the manuscript.

\section{FUNDING}

This study was supported by grants number 14158 and 15918 from the Jubilaeumsfonds of the Austrian National Bank (KR).

\section{ACKNOWLEDGMENTS}

We want to thank all the referring colleagues and especially the NEMOS group for their help and support with this study!

\section{SUPPLEMENTARY MATERIAL}

The Supplementary Material for this article can be found online at: https://www.frontiersin.org/articles/10.3389/fneur. 2020.00415/full\#supplementary-material

Supplementary Table 1 | Demographic, serologic, clinical, radiologic and therapeutic characteristics of the included patients. 
12. Wingerchuk DM, Banwell B, Bennett JL, Cabre P, Carroll W, Chitnis T, et al. International consensus diagnostic criteria for neuromyelitis optica spectrum disorders. Neurology. (2015) 85:177-89. doi: 10.1212/wnl.00000000000 01729

13. Waters P, Reindl M, Saiz A, Schanda K, Tuller F, Kral V, et al. Multicentre comparison of a diagnostic assay: aquaporin-4 antibodies in neuromyelitis optica. J Neurol Neurosurg Psychiatr. (2016) 87:1005-15. doi: 10.1136/jnnp-2015-312601

14. Jarius S, Franciotta D, Bergamaschi R, Wright H, Littleton E, Palace J, et al. NMO-IgG in the diagnosis of neuromyelitis optica. Neurology. (2007) 68:1076-7. doi: 10.1212/01.wnl.0000256822.01222.bd

15. Jarius S, Probst C, Borowski K, Franciotta D, Wildemann B, Stoecker W, et al. Standardized method for the detection of antibodies to aquaporin- 4 based on a highly sensitive immunofluorescence assay employing recombinant target antigen. J Neurol Sci. (2010) 291:52-6. doi: 10.1016/j.jns.2010.01.002

16. Jarius S, Wildemann B. Aquaporin-4 antibodies (NMO-IgG) as a serological marker of neuromyelitis optica: a critical review of the literature. Brain Pathol. (2013) 23:661-83. doi: 10.1111/bpa.12084

17. Hamid SH, Elsone L, Mutch K, Solomon T, Jacob A. The impact of 2015 neuromyelitis optica spectrum disorders criteria on diagnostic rates. Mult Scler. (2017) 23:228-33. doi: 10.1177/1352458516663853

18. Sepulveda M, Aldea M, Escudero D, Llufriu S, Arrambide G, Otero-Romero S, et al. Epidemiology of NMOSD in Catalonia: Influence of the new 2015 criteria in incidence and prevalence estimates. Mult Scler. (2017) 1:1352458517735191. doi: 10.1177/1352458517735191

19. Mader S, Gredler V, Schanda K, Rostasy K, Dujmovic I, Pfaller K, et al. Complement activating antibodies to myelin oligodendrocyte glycoprotein in neuromyelitis optica and related disorders. J Neuroinflamm. (2011) 8:184. doi: 10.1186/1742-2094-8-184

20. Kitley J, Waters P, Woodhall M, Leite MI, Murchison A, George J, et al. Neuromyelitis optica spectrum disorders with aquaporin-4 and myelinoligodendrocyte glycoprotein antibodies: a comparative study. JAMA Neurol. (2014) 71:276-83. doi: 10.1001/jamaneurol.2013.5857

21. Sato DK, Callegaro D, Lana-Peixoto MA, Waters PJ, de Haidar Jorge FM, Takahashi T, et al. Distinction between MOG antibody-positive and AQP4 antibody-positive NMO spectrum disorders. Neurology. (2014) 82:474-81. doi: 10.1212/wnl.0000000000000101

22. Probstel AK, Rudolf G, Dornmair K, Collongues N, Chanson JB, Sanderson NS, et al. Anti-MOG antibodies are present in a subgroup of patients with a neuromyelitis optica phenotype. J Neuroinflammation. (2015) 12:46. doi: 10.1186/s12974-015-0256-1

23. Lechner C, Baumann M, Hennes EM, Schanda K, Marquard K, Karenfort M, et al. Antibodies to MOG and AQP4 in children with neuromyelitis optica and limited forms of the disease. J Neurol Neurosurg Psychiatr. (2016) 87:897-905. doi: 10.1136/jnnp-2015-311743

24. Hacohen Y, Absoud M, Deiva K, Hemingway C, Nytrova P, Woodhall M, et al. Myelin oligodendrocyte glycoprotein antibodies are associated with a nonMS course in children. Neurol Neuroimmunol Neuroinflamm. (2015) 2:e81. doi: 10.1212/nxi.0000000000000081

25. Jarius S, Ruprecht K, Kleiter I, Borisow N, Asgari N, Pitarokoili K, et al. MOGIgG in NMO and related disorders: a multicenter study of 50 patients. part 2: epidemiology, clinical presentation, radiological and laboratory features, treatment responses, and long-term outcome. J Neuroinflamm. (2016) 13:280. doi: 10.1186/s12974-016-0718-0

26. Jarius S, Ruprecht K, Kleiter I, Borisow N, Asgari N, Pitarokoili K, et al. MOG-IgG in NMO and related disorders: a multicenter study of 50 patients. Part 1: frequency, syndrome specificity, influence of disease activity, long-term course, association with AQP4-IgG, and origin. J Neuroinflamm. (2016) 13:279. doi: 10.1186/s12974-0160717-1

27. Hennes EM, Baumann M, Schanda K, Anlar B, Bajer-Kornek B, Blaschek A, et al. Prognostic relevance of MOG antibodies in children with an acquired demyelinating syndrome. Neurology. (2017) 89:900-8. doi: 10.1212/wnl.0000000000004312

28. Hacohen Y, Banwell B. Treatment approaches for MOG-Ab-associated demyelination in children. Curr Treat Options Neurol. (2019) 21:2. doi: 10.1007/s11940-019-0541-x
29. Fragoso YD, Ferreira ML, Oliveira EM, Domingues RB, Ribeiro TA, Brooks JB, et al. Neuromyelitis optica with onset in childhood and adolescence. Pediatr Neurol. (2014) 50:66-8. doi: 10.1016/j.pediatrneurol.2013.07.003

30. Absoud M, Lim MJ, Appleton R, Jacob A, Kitley J, Leite MI, et al. Paediatric neuromyelitis optica: clinical, MRI of the brain and prognostic features. $J$ Neurol Neurosurg Psychiatr. (2015) 86:470-2. doi: 10.1136/jnnp-2014-308550

31. Chitnis T, Ness J, Krupp L, Waubant E, Hunt T, Olsen CS, et al. Clinical features of neuromyelitis optica in children: US network of pediatric MS Centers report. Neurology. (2016) 86:245-52. doi: 10.1212/wnl.0000000000002283

32. Gombolay GY, Chitnis T. Pediatric Neuromyelitis Optica Spectrum Disorders. Curr Treat Options Neurol. (2018) 20:19. doi: 10.1007/s11940-018-0502-9

33. Houzen $H$, Niino M, Hirotani M, Fukazawa T, Kikuchi S, Tanaka $\mathrm{K}$, et al. Increased prevalence, incidence, and female predominance of multiple sclerosis in northern Japan. J Neurol Sci. (2012) 323:117-22. doi: 10.1016/j.jns.2012.08.032

34. Jacob A, Panicker J, Lythgoe D, Elsone L, Mutch K, Wilson M, et al. The epidemiology of neuromyelitis optica amongst adults in the Merseyside county of United Kingdom. J Neurol. (2013) 260:2134-7. doi: 10.1007/s00415-013-6926-y

35. Etemadifar M, Dashti M, Vosoughi R, Abtahi SH, Ramagopalan SV, Nasr Z. An epidemiological study of neuromyelitis optica in Isfahan. Mult Scler. (2014) 20:1920-2. doi: 10.1177/1352458514537699

36. Pandit L, Kundapur R. Prevalence and patterns of demyelinating central nervous system disorders in urban Mangalore, South India. Mult Scler. (2014) 20:1651-3. doi: 10.1177/1352458514521503

37. Etemadifar M, Nasr Z, Khalili B, Taherioun M, Vosoughi R. Epidemiology of neuromyelitis optica in the world: a systematic review and meta-analysis. Mult Scler Int. (2015) 2015:174720. doi: 10.1155/2015/174720

38. Alvarenga MP, Schimidt S, Alvarenga RP. Epidemiology of neuromyelitis optica in Latin America. Mult Scler J Exp Transl Clin. (2017) 3:2055217317730098. doi: 10.1177/2055217317730098

39. Bukhari W, Prain KM, Waters P, Woodhall M, O'Gorman CM, Clarke L, et al. Incidence and prevalence of NMOSD in Australia and New Zealand. J Neurol Neurosurg Psychiatr. (2017) 88:632-8. doi: 10.1136/jnnp-2016-3 14839

40. Hyun JW, Jeong IH, Joung A, Kim SH, Kim HJ. Evaluation of the 2015 diagnostic criteria for neuromyelitis optica spectrum disorder. Neurology. (2016) 86:1772-9. doi: 10.1212/wnl.00000000000 02655

41. Langer-Gould A, Zhang JL, Chung J, Yeung Y, Waubant E, Yao J. Incidence of acquired CNS demyelinating syndromes in a multiethnic cohort of children. Neurology. (2011) 77:1143-8. doi: 10.1212/WNL.0b013e31822facdd

42. Boesen MS, Magyari M, Born AP, Thygesen LC. Pediatric acquired demyelinating syndromes: a nationwide validation study of the Danish national patient register. Clin Epidemiol. (2018) 10:391-9. doi: $10.2147 /$ clep.S156997

43. Dahan A, Brilot F, Leventer R, Kornberg AJ, Dale RC, Yiu EM. Neuromyelitis optica spectrum disorder and anti-aquaporin 4 channel immunoglobulin in an australian pediatric demyelination cohort. J Child Neurol. (2020) 35:291-6. doi: 10.1177/0883073819895191

44. Boesen MS, Jensen PEH, Born AP, Magyari M, Nilsson AC, Hoei-Hansen $\mathrm{C}$, et al. Incidence of pediatric neuromyelitis optica spectrum disorder and myelin oligodendrocyte glycoprotein antibody-associated disease in Denmark 20082018: A nationwide, population-based cohort study. Mult Scler Relat Disord. (2019) 33:162-7. doi: 10.1016/j.msard.2019.06.002

45. Trebst C, Jarius S, Berthele A, Paul F, Schippling S, Wildemann B, et al. Update on the diagnosis and treatment of neuromyelitis optica: recommendations of the neuromyelitis optica study group. (NEMOS). J Neurol. (2014) 261:1-16. doi: 10.1007/s00415-013-7169-7

46. Mader S, Lutterotti A, Di Pauli F, Kuenz B, Schanda K, Aboul-Enein F, et al. Patterns of antibody binding to aquaporin-4 isoforms in neuromyelitis optica. PLoS ONE. (2010) 5:e10455. doi: 10.1371/journal.pone.0010455

47. Reindl M, Schanda K, Woodhall M, Tea F, Ramanathan S, Sagen $\mathrm{J}$, et al. International multicenter examination of MOG antibody assays. Neurol Neuroimmunol Neuroinflamm. (2020) 7:e674. doi: $10.1212 /$ nxi.0000000000000674 
48. Reindl M, Waters P. Myelin oligodendrocyte glycoprotein antibodies in neurological disease. Nat Rev Neurol. (2019) 15:89-102. doi: 10.1038/s41582-018-0112-x

49. Rostasy K, Mader S, Hennes EM, Schanda K, Gredler V, Guenther A, et al. Persisting myelin oligodendrocyte glycoprotein antibodies in aquaporin-4 antibody negative pediatric neuromyelitis optica. Mult Scler. (2013) 19:10529. doi: $10.1177 / 1352458512470310$

50. Hoftberger R, Sepulveda M, Armangue T, Blanco Y, Rostasy K, Calvo AC, et al. Antibodies to MOG and AQP4 in adults with neuromyelitis optica and suspected limited forms of the disease. Mult Scler. (2015) 21:866-74. doi: $10.1177 / 1352458514555785$

51. Borisow N, Mori M, Kuwabara S, Scheel M, Paul F. Diagnosis and treatment of NMO spectrum disorder and MOG-encephalomyelitis. Front Neurol. (2018) 9:888. doi: 10.3389/fneur.2018.00888

52. Di Pauli F, Berger T. Myelin Oligodendrocyte glycoprotein antibodyassociated disorders: toward a new spectrum of inflammatory demyelinating CNS disorders? Front Immunol. (2018) 9:2753. doi: 10.3389/fimmu.2018.02753

53. Hennes EM, Baumann M, Lechner C, Rostasy K. MOG Spectrum disorders and role of MOG-antibodies in clinical practice. Neuropediatrics. (2018) 49:3-11. doi: 10.1055/s-0037-1604404

54. Ungureanu A, de Seze J, Ahle G, Sellal F. Myelin oligodendrocyte glycoprotein antibodies in neuromyelitis optica spectrum disorder. Rev Neurol. (2018) 174:675-9. doi: 10.1016/j.neurol.2018.01.378

55. Fujihara K. Neuromyelitis optica spectrum disorders: still evolving and broadening. Curr Opin Neurol. (2019) 32:385-94. doi: $10.1097 /$ wco. 0000000000000694

56. Jurynczyk M, Jacob A, Fujihara K, Palace J. Myelin oligodendrocyte glycoprotein (MOG) antibody-associated disease: practical considerations. Pract Neurol. (2019) 19:187-95. doi: 10.1136/practneurol-2017-001787

57. Banwell B, Tenembaum S, Lennon VA, Ursell E, Kennedy J, BarOr A, et al. Neuromyelitis optica-IgG in childhood inflammatory demyelinating CNS disorders. Neurology. (2008) 70:344-52. doi: 10.1212/01.wnl.0000284600.80782.d5
58. Lotze TE, Northrop JL, Hutton GJ, Ross B, Schiffman JS, Hunter JV. Spectrum of pediatric neuromyelitis optica. Pediatrics. (2008) 122:e1039-47. doi: 10.1542/peds.2007-2758

59. Huppke P, Bluthner M, Bauer O, Stark W, Reinhardt K, Huppke B, et al. Neuromyelitis optica and NMO-IgG in European pediatric patients. Neurology. (2010) 75:1740-4. doi: 10.1212/WNL.0b013e3181 fc2823

60. Kunchok A, Chen JJ, McKeon A, Mills JR, Flanagan EP, Pittock SJ. Coexistence of myelin oligodendrocyte glycoprotein and aquaporin-4 antibodies in adult and pediatric patients. JAMA Neurol. (2019) 77:257-9. doi: 10.1001/jamaneurol.2019.3656

61. Duignan S, Wright S, Rossor T, Cazabon J, Gilmour K, Ciccarelli O, et al. Myelin oligodendrocyte glycoprotein and aquaporin-4 antibodies are highly specific in children with acquired demyelinating syndromes. Dev Med Child Neurol. (2018) 60:958-62. doi: 10.1111/dmcn.13703

Conflict of Interest: MBr, EW, KS, and MBa report no disclosures related to the present work. KR received speakers' honoraria from Novartis and served on the advisory board of the PARADIGM study. MR receives payments for antibody assays (MOG, AQP4, and other autoantibodies) and for MOG and AQP4 antibody validation experiments organized by Euroimmun (Lübeck, Germany). CL served as a consultant for Roche. BK received speakers' honoraria and travel support and served as advisor for Biogen, Celgene, Merck, Novartis, Sanofi-Genzyme, Roche, and TEVA.

The handling editor declared a past co-authorship with one of the authors, MR.

Copyright (๑) 2020 Lechner, Breu, Wendel, Kornek, Schanda, Baumann, Reindl and Rostásy. This is an open-access article distributed under the terms of the Creative Commons Attribution License (CC BY). The use, distribution or reproduction in other forums is permitted, provided the original author(s) and the copyright owner $(s)$ are credited and that the original publication in this journal is cited, in accordance with accepted academic practice. No use, distribution or reproduction is permitted which does not comply with these terms. 